\title{
GENETIC DIVERSITY OF CROCUS SATIVUS AND ITS CLOSE RELATIVE SPECIES ANALYZED BY iPBS-RETROTRANSPOSONS
}

\author{
Aysun GEDIK ${ }^{1 *}$, Duygu ATES ${ }^{2 *}$, Semih ERDOGMUS ${ }^{2}$, Gonul COMERTPAY ${ }^{3}$, Muhammed Bahattin \\ TANYOLAC $C^{2 * *}$ Hakan OZKAN $N^{1,4^{* *}}$ \\ ${ }^{1}$ Cukurova University, Department of Biotechnology, Institute of Natural and Applied Sciences, Adana, \\ TURKEY \\ ${ }^{2}$ Ege University, Department of Bioengineering, Izmir, TURKEY \\ ${ }^{3}$ Eastern Mediterranean Agricultural Research Institute, Adana, TURKEY \\ ${ }^{4}$ Cukurova University, Faculty of Agriculture Department of Field Crops, Adana, TURKEY \\ *: These authors contributed equally to this article \\ **Corresponding authors: bahattin.tanyolac@gmail.com, hozkan@cu.edu.tr
}

Received: 18.10.2017

\begin{abstract}
Saffron (Crocus $\mathrm{L}$.) is a member of Crocoideae, the biggest of four subfamilies in the Iridaceae family. It has $2 n=3 x=24$ chromosomes and is triploid; thus, it is sterile. In previous research, different molecular DNA markers were used but molecular characterization and genetic diversity of this complex genus have not yet been clarified. Therefore, current study aimed to determine the molecular characterization of saffron and its close relative species using inter-primer binding site (iPBS)-retrotransposon markers. Eighty-three iPBSretrotransposon primers were used in $28 \mathrm{C}$. sativus genotypes and 17 close relative species of saffron to identify their genetic diversity. Sixteen polymorphic iPBS-retrotransposon primers generated a total of 401 polymorphic scorable bands. The mean PIC value, Nei's genetic diversity and Shannon's information index (I) were calculated as $0.85,0.16$ and 0.29 , respectively. The results of the Unweighted Pair Group Method with Arithmetic mean UPGMA dendrogram and Principal Coordinates Analysis PCoA analysis indicated a spatial representation of the relative genetic distances among 28 saffron samples and the 17 close relative species were categorized under two distinct groups. Saffron genotypes showed very limited genetic variation and according to the iPBS-retrotransposon data, its close relatives were $C$. cartwrightianus and $C$. pallasii subsp. pallasii.
\end{abstract}

Keywords: Crocus sativus, genetic diversity, inter-primer binding site retrotransposons, saffron.

\section{INTRODUCTION}

Crocus L. is a member of Crocoideae, the biggest of presently known four subfamilies in the Iridaceae family (Busconi et al., 2015; Goldblatt et al., 2006). While this genus was originally considered to contain approximately 90 small species (Petersen et al., 2008), molecular, morphological and karyological studies undertaken in recent years (Kerndorff et al., 2012; Kerndorff et al., 2013; Harpke et al., 2013; Schneider et al., 2012) have shown that the number of Crocus species is presumably higher than 160 (Harpke et al., 2015) and these species are found in a wide range of habitats, including meadows, scrub and woodland (Rubio-Moraga et al., 2009). The majority of Crocus species and subspecies are naturally grown in the Balkan Peninsula, Greek, and Turkey (Petersen et al., 2008; Kerndorff and Pasche, 2011; Kerndorff et al., 2012; Peruzzi and Carta, 2011). Among all Crocus diversity in the world, Turkey's flora includes a total of 132 Crocus species (108 endemic) with different flowering times (Mathew, 1982; Ozhatay, 2002; Kerndorff and Pasche, 2004; Arslan et al., 2007; AlaviKia et al., 2008; Yuzbasioglu et al., 2015).

Crocus L. is primarily known for the commercially cultivated species $C$. sativus, generally called "saffron", which is one of the most consumed spices in the world (Negbi, 2003). Saffron and its efficacious components have many applications in many industries such as textile (Mortazavi et al., 2012), perfume (Mousavi and Bathaie, 2011), food (Babaei et al., 2014) and pharmaceuticals (Hadizadeh et al., 2010). In addition, saffron is used as an enhancer of learning ability (Pitsikas and Sakellaridis, 2006), antidepressant (Basti et al., 2007), anti-cancer agent (Abdullaev, 2002; Fernández, 2006), and antioxidant component (Verma and Bordia, 1998). The retail price of saffron can reach 20.000 Euro/kg; thus making it one of the most expensive spices in the world 
(Busconi et al., 2015). Due to its great commercial importance, the exploration of the genetic diversity of saffron and relationships between its accessions are significant for germplasm conservation and breeding strategies.

Saffron has $2 n=3 x=24$ chromosomes and is triploid; thus, it is sterile (Gribbon et al., 1999). However, the sterility of this species has limited the application of conventional genetic diversity strategies (Busconi et al., 2015). In the last four decades, classical strategies have been complemented with morphological markers to determine the genetic diversity of saffron; however, DNA markers have the potential to provide more reliable results than morphological markers in genetic diversity research since they represent the variation at the DNA level and are not affected by different environmental conditions (Mondini et al., 2009; Weising et al., 2005; Kumar, 1999; Kumar et al., 2009). Despite this potential, in the literature, there are only few articles reporting on the determination and classification of the systematic and genetic diversity of saffron (Frello and Heslop-Harrison, 2000; Nørbæk et al., 2002; Castillo et al., 2005; Frizzi et al., 2007; Petersen et al., 2008; Seberg and Petersen, 2009; Moraga et al., 2010). These studies have employed different molecular DNA markers such as inter simple sequence repeat (ISSR) (Rubio-Moraga et al., 2009), random amplified polymorphic DNA (RAPD) (RubioMoraga et al., 2009), sequence-related amplified polymorphism (SRAP) (Babaei et al., 2014), amplified fragment length polymorphism (AFLP) (Busconi et al., 2015), simple sequence repeats (SSR) (Mir et al., 2015) and inter-retroelement amplified polymorphism (IRAP) (Alsayied et al., 2015; Alavi-Kia et al., 2008) markers but have not fully elucidated the molecular characterization and genetic diversity of this complex genus. Alsayied et al. (2015) reported that according to the IRAP data, $C$. sativus had minimum genetic variation and its ancestors were $C$. cartwrightianus and $C$. pallasii subsp. pallasii.

Retrotransposons are mobile genetic elements that generally constitute $50 \%$ of plant genome (may reach as high as $90 \%$ ) and play a significant role in the formation of genetic diversity (SanMiguel et al., 1996; Pearce et al., 1996; Schulman et al., 2012). In addition, retrotransposons are very useful for the development of new markers because of their high prevalence and genome-specific distribution (Schulman et al., 2012). To date, several types of retrotransposons such as retrotransposon-microsatellite and amplified polymorphism (REMAP), IRAP and retrotransposon-based insertion polymorphism (RBIP) have been used for research on plant characterization (Kalendar et al., 2011; Schulman et al., 2012; Alavi-Kia et al., 2008; Alsayied et al., 2015). However, the process of developing retrotransposon markers for a new plant species has certain restricting factors such as the variation of the polymerase chain reaction (PCR) product size and the necessity of cloning and sequence information to design primers that match the flanking genomic DNA at each specific site (Kalendar et al., 2010). Recently, to reduce the negative effect of these factors, Kalendar et al. (2010) proposed a new highly effective and universal molecular marker system based on the conversed sequences of retrotransposons, called "inter-primer binding site (iPBS) retrotransposons". In particular, the amplification technique of the iPBS-retrotransposons is very practical and comprises a powerful DNA fingerprinting technology that does not require prior sequence information. Thus, reproducibility is very high owing to the primer length and strong stringency success by selecting appropriate annealing temperature (Guo et al., 2014). The iPBS-retrotransposon marker system has been successfully implemented in phylogenetic studies, clone identification research, and genetic diversity analyses (Smýkal et al., 2011; Baránek et al., 2012; Gailite and Rungis, 2012). To date, genetic diversity evaluation of certain plant species such as flax (Smýkal et al., 2011), apricot (Baránek et al., 2012), Saussurea (Gailite and Rungis, 2012), chickpea (Andeden et al., 2013), rice (Comertpay et al., 2015), lentil (Baloch et al., 2015b) and pea (Baloch et al., 2015a) have been studied using iPBSretrotransposons; however, to the best of our knowledge, the current study is the first to determine the molecular characterization of saffron and its close relative species using this marker system.

\section{MATERIALS AND METHODS}

\section{Plant materials and DNA extraction}

In the current study, a total of 28 C. sativus samples collected in Turkey, Iran, Spain, Azerbaijan, Italy, France and Greek and 17 close relative species of saffron collected in Turkey and Greek were used as plant material. Table 1 presents the number of genotypes, taxon and collection site of each $C$. sativus sample and Table 2 gives information on the close relative species of these samples. 
Table 1. List of C. sativus samples used for molecular characterization analysis.

\begin{tabular}{|c|c|c|c|}
\hline Genotype number & Genotype name & Taxon & Collection site \\
\hline 1 & Tokat 1 & C. sativus & Tokat / Turkey \\
\hline 2 & Tokat 2 & C. sativus & Tokat / Turkey \\
\hline 3 & Tokat 3 & C. sativus & Tokat / Turkey \\
\hline 4 & Tokat 4 & C. sativus & Tokat / Turkey \\
\hline 5 & Tokat 5 & C. sativus & Tokat / Turkey \\
\hline 6 & Iranian 1 & C. sativus & Iranian \\
\hline 7 & Iranian 2 & C. sativus & Iranian \\
\hline 8 & Iranian 3 & C. sativus & Iranian \\
\hline 9 & Iranian 4 & C. sativus & Iranian \\
\hline 10 & Iranian 5 & C. sativus & Iranian \\
\hline 11 & AHBR & C. sativus & Kastamonu / Turkey \\
\hline 12 & 49 & C. sativus & Kastamonu / Turkey \\
\hline 13 & 50 & C. sativus & Kastamonu / Turkey \\
\hline 14 & 51 & C. sativus & Kastamonu / Turkey \\
\hline 15 & 52 & C. sativus & Kastamonu / Turkey \\
\hline 16 & 53 & C. sativus & Kastamonu / Turkey \\
\hline 17 & 54 & C. sativus & Kastamonu / Turkey \\
\hline 18 & 61 & C. sativus & Kastamonu / Turkey \\
\hline 19 & 62 & C. sativus & Kastamonu / Turkey \\
\hline 20 & $\mathrm{AABB}$ & C. sativus & Kastamonu / Turkey \\
\hline 21 & BCV 001584 & C. sativus & Spain \\
\hline 22 & BCV 001687 & C. sativus & Iranian \\
\hline 23 & BCV 001783 & C. sativus & Azerbaijan \\
\hline 24 & BCV 001806 & C. sativus & Italy \\
\hline 25 & BCV 002708 & C. sativus & France \\
\hline 26 & BCV 002911 & C. sativus & Greek \\
\hline 27 & 5 & C. sativus & Kastamonu / Turkey \\
\hline 28 & SC77 & C. sativus & Kastamonu / Turkey \\
\hline
\end{tabular}

Table 2. List of close relative species of saffron samples used for molecular characterization analysis.

\begin{tabular}{llll}
\hline $\begin{array}{l}\text { Genotype } \\
\text { number }\end{array}$ & $\begin{array}{l}\text { Genotype } \\
\text { name }\end{array}$ & Taxon & Collection site \\
\hline 29 & SC2 & C. pallasii subsp. pallasii & Mugla / Turkey \\
30 & SC5 & C. pallasii subsp. pallasii & Mugla / Turkey \\
31 & SC1 & C. pallasii subsp. pallasii & Mugla / Turkey \\
32 & SC8 & C. pallasii subsp. pallasii & Mugla / Turkey \\
33 & SC10 & C. pallasii subsp. pallasii & Izmir / Turkey \\
34 & SC12 & C. pallasii subsp. pallasii & Denizli / Turkey \\
35 & SC17 & C. pallasii subsp. pallasii & Konya / Turkey \\
36 & SC18 & C. pallasii subsp. pallasii & Konya / Turkey \\
37 & BZDG & C. pallasii subsp. pallasii & Izmir / Turkey \\
38 & DDGL & C. pallasii subsp. pallasii & Isparta / Turkey \\
39 & SB60 & C. pallasii x C. mathewi & Antalya / Turkey \\
40 & SC9 & C. mathewii & Antalya / Turkey \\
41 & SC20 & C. pallasii subsp. dispathaceus & Mersin / Turkey \\
42 & SC21 & C. pallasii subsp. dispathaceus & Mersin / Turkey \\
43 & SC22 & C. pallasii subsp. turcicus & Gaziantep / Turkey \\
44 & SC45 & C. asumaniae & Antalya / Turkey \\
45 & CRTW & C. cartwrightianus & Greek \\
\hline
\end{tabular}

The total genomic DNA was extracted from all samples using the method described by Doyle and Doyle (1987), with some minor modifications (Kafkas, 2006). Then, the purity of the genomic DNA was visually checked on agarose gel $(1 \%)$ and total DNA concentration was measured using a Qubit®2.0 Fluorometer (Life
Technologies, US). The final DNA concentration was adjusted to $5 \mathrm{ng} / \mu \mathrm{L}$ to use in PCR.

\section{Analysis of iPBS-retrotransposon markers}

In current study, 83 iPBS-retrotransposon primers developed by Kalendar et al. (2010) were used. DNA 
amplification was performed according to the protocol of Andeden et al. (2013). PCR amplifications were carried out using $25 \mu \mathrm{l}$ reaction mixture containing $5 \mathrm{ng}$ template DNA, 3 x Dream Taq Green PCR buffer (Fermentas), 10 $\mu \mathrm{M}$ of primer (single primer), $5 \mathrm{mM} \mathrm{dNTPs}$, and 1.75-unit Dream Taq DNA polymerase (Fermentas).

The thermal cycling profile of the PCR was as follows: initial denaturation at $95^{\circ} \mathrm{C}$ for $4 \mathrm{~min}, 30$ cycles of $95{ }^{\circ} \mathrm{C}$ for $15 \mathrm{~s}, 50-65{ }^{\circ} \mathrm{C}$ for $1 \mathrm{~min}$ and $68{ }^{\circ} \mathrm{C}$ for $1 \mathrm{~min}$; and final extension at $72{ }^{\circ} \mathrm{C}$ for $5 \mathrm{~min}$ (Kalendar et al., 2010). For the separation of all the PCR products, 1.7-2 \% (w/v) agarose gel electrophoresis was used with $1 \mathrm{x}$ TBE buffer for $2 \mathrm{~h}$, stained with ethidium bromide and visualized by a G-BOX gel documentation system (Syngene, USA).

\section{Scoring and analysis of the data}

The DNA bands of each iPBS-retrotransposon primer were manually scored. Only the clear and strong bands were considered and the faint bands were ignored to exclude artificial bands. To build binary matrices, the presence or absence of an iPBS-retrotransposon band at a particular locus was scored as " 1 " and " 0 ", respectively. The polymorphism information content (PIC) for dominant iPBS-retrotransposon markers was calculated by the formula described by de Riek et al. (2001) and Baloch et al. (2015a);

$$
\mathrm{PIC}_{\mathrm{i}}=2 \mathrm{f}_{\mathrm{i}}\left(1-\mathrm{f}_{\mathrm{i}}\right)
$$

where " $f_{i}$ " is the band present (amplified allele) frequency of the marker and " $\left(1-\mathrm{f}_{\mathrm{i}}\right)$ " refers to the band absent (null allele) frequency of the marker. The NTSYSpc 2.11 V software package (Rohlf, 2000) was used for the analysis of the iPBS binary matrices. Dendrograms were constructed based on Dice's genetic similarity coefficient ( $\mathrm{Nei}$ and $\mathrm{Li}, 1979$ ) using the unweighted pair-group method with arithmetic averages (UPGMA). To calculate Nei's distance coefficient (h), the Splits Tree4 software package (Huson and Bryant, 2006) was used on the binary data and then a Neighbor-Net tree (Nei, 1987) was drawn. Shannon's information index (I) was calculated using the $\mathrm{R}$ computer program.

\section{RESULTS}

\section{iPBS-retrotransposon marker system}

Eighty-three iPBS-Retrotransposon primers were tested on 4 saffron genotypes for initial screening. Of these, 16 primers represented (19\%) a large number of good and clear band profiles. Therefore, these 16 primers yielding reproducible polymorphic bands were selected for the amplifications of all 28 saffron samples and its 17 close relative species. The 16 iPBS-retrotransposon primers generated a total of 401 polymorphic scorable bands (Table 3 ). The number of polymorphic bands per primer ranged from 15 (iPBS 2238) to 35 (iPBS 2081), with an average of 25 bands per primer (Table 3 ).

Table 3. Information about the iPBS-Retrotransposon primers used in current study.

\begin{tabular}{clcccccc}
\hline $\begin{array}{c}\text { Primer } \\
\text { Number }\end{array}$ & Primer Name & NTB & NSPB & NSPB \% & PIC & h & I \\
\hline 1 & iPBS 2075 & 25 & 25 & 6.23 & 0.85 & 0.20 & 0.36 \\
2 & iPBS 2079 & 36 & 34 & 8.48 & 0.86 & 0.12 & 0.22 \\
3 & iPBS 2081 & 35 & 35 & 8.73 & 0.89 & 0.15 & 0.30 \\
4 & iPBS 2085 & 21 & 19 & 4.74 & 0.82 & 0.19 & 0.31 \\
5 & iPBS 2095 & 26 & 24 & 5.98 & 0.73 & 0.23 & 0.33 \\
6 & iPBS 2219 & 22 & 22 & 5.49 & 0.90 & 0.12 & 0.25 \\
7 & iPBS 2229 & 28 & 28 & 6.98 & 0.87 & 0.14 & 0.28 \\
8 & iPBS 2238 & 15 & 15 & 3.74 & 0.79 & 0.17 & 0.25 \\
9 & iPBS 2239 & 26 & 26 & 6.48 & 0.82 & 0.20 & 0.34 \\
10 & iPBS 2273 & 21 & 20 & 4.99 & 0.92 & 0.10 & 0.21 \\
11 & iPBS 2375 & 23 & 23 & 5.73 & 0.84 & 0.16 & 0.26 \\
12 & iPBS 2376 & 25 & 25 & 6.23 & 0.88 & 0.15 & 0.28 \\
13 & iPBS 2381 & 31 & 30 & 7.48 & 0.82 & 0.18 & 0.28 \\
14 & iPBS 2400 & 26 & 26 & 6.48 & 0.88 & 0.12 & 0.23 \\
15 & iPBS 2401 & 30 & 28 & 6.98 & 0.84 & 0.18 & 0.31 \\
16 & iPBS 2415 & 23 & 21 & 5.24 & 0.83 & 0.19 & 0.37 \\
\hline Total & - & 413 & 401 & - & - & - & - \\
Average & - & - & - & - & 0.85 & 0.16 & 0.29 \\
\hline
\end{tabular}

NTB; number of total bands. NSPB; number of scorable polymorphic bands.

PIC; polymorphism information index. h; Nei's gene diversity. I; Shannon's information index.

The PIC value was used to calculate the effectiveness of polymorphic loci in defining genetic diversity among all genotypes. The mean PIC value was determined as 0.85, ranging from 0.73 (iPBS 2095) to 0.92 (iPBS 2273) (Table 3) indicating that the iPBSretrotransposon primers had the potential to develop high loci polymorphism. 


\section{Genetic diversity between saffron and its close relative species}

The data on each iPBS-retrotransposon was interpreted using the following statistical parameters; PIC, Nei's genetic diversity (h), and Shannon's information index (I). These parameters were evaluated for all the genotypes as a single population. Nei's genetic diversity among saffron genotypes and its close relative species varied from 0.10 (iPBS 2273) to 0.23 (iPBS 2095), with a mean value of 0.16 and Shannon's information index (I) varied from 0.21 (iPBS 2273) to 0.37 (iPBS 2415), with a mean value of 0.29 (Table 3 ).

The iPBS-retrotransposon analysis predicted that the highest genetic variation was between CRTW and SC9 while the lowest genetic variation was between BCV
001687, BCV 001584, BCV 001783, BCV 001806, and BCV 002911.

\section{Cluster and principal component analysis for the iPBS-retrotransposon markers}

According to the results of the unweighted pair group analysis with the arithmetic mean (UPGMA) dendrogram including a heat map, 28 saffron samples and its 17 close relative species were under two main clusters (Figures 1 and 2). The first cluster contained 28 saffron genotypes as listed in Table 1 and the second cluster comprised 17 close relative species as shown in Table 2. This indicates that there was a good differentiation between the saffron genotypes and its close relative species. The two main groups were further divided into different subgroups with very low variability.

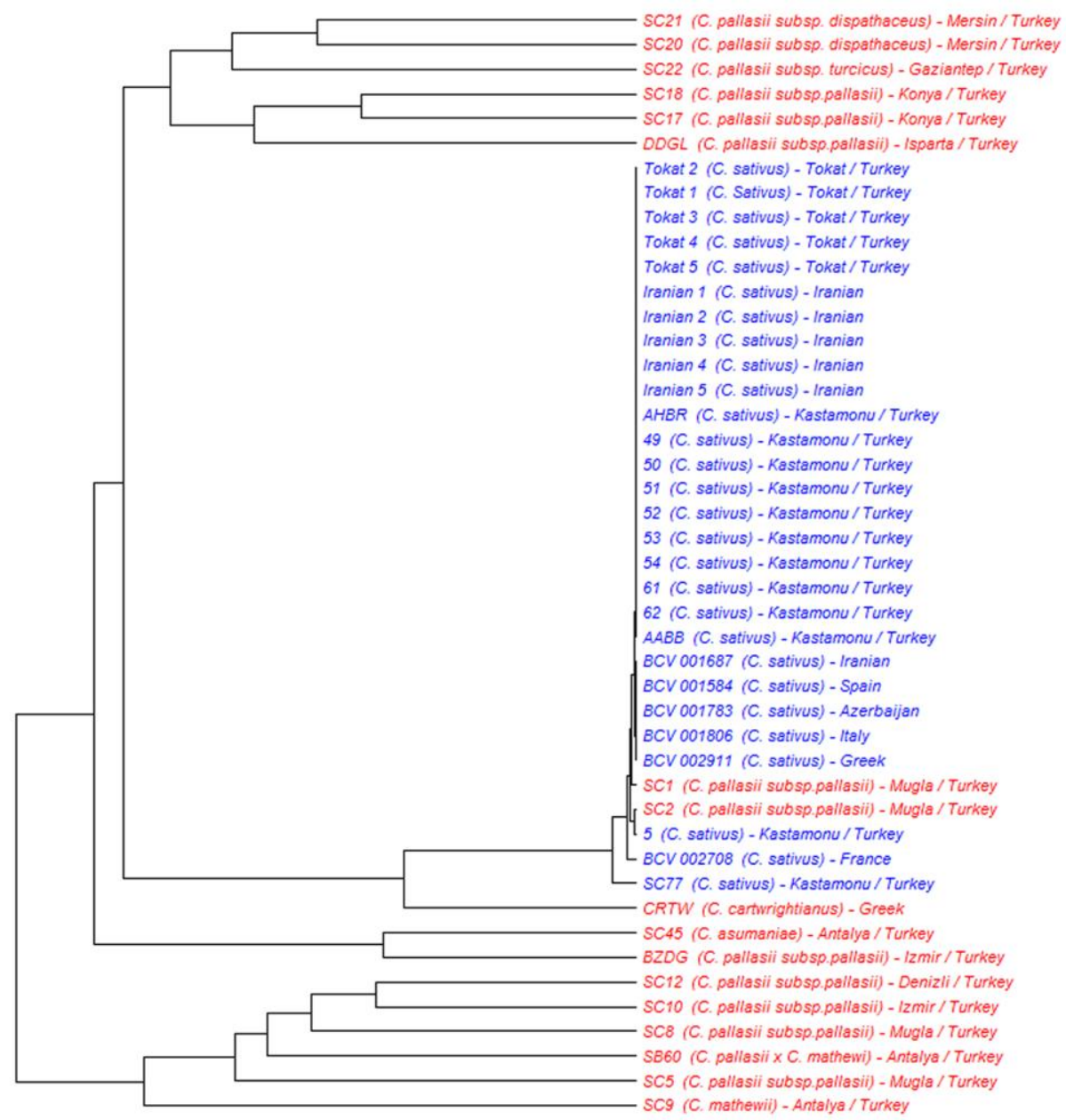

Figure 1. UPGMA dendrogram of 45 saffron genotypes and its close relative species based on iPBS-retrotransposon primers.

The results of the principal component analysis indicated two distinct groups in the spatial representation of the relative genetic distances among 28 saffron samples and its 17 close relative species (Figure 3), supporting the results presented in the UPGMA dendrogram (Figures 1 and 2). 


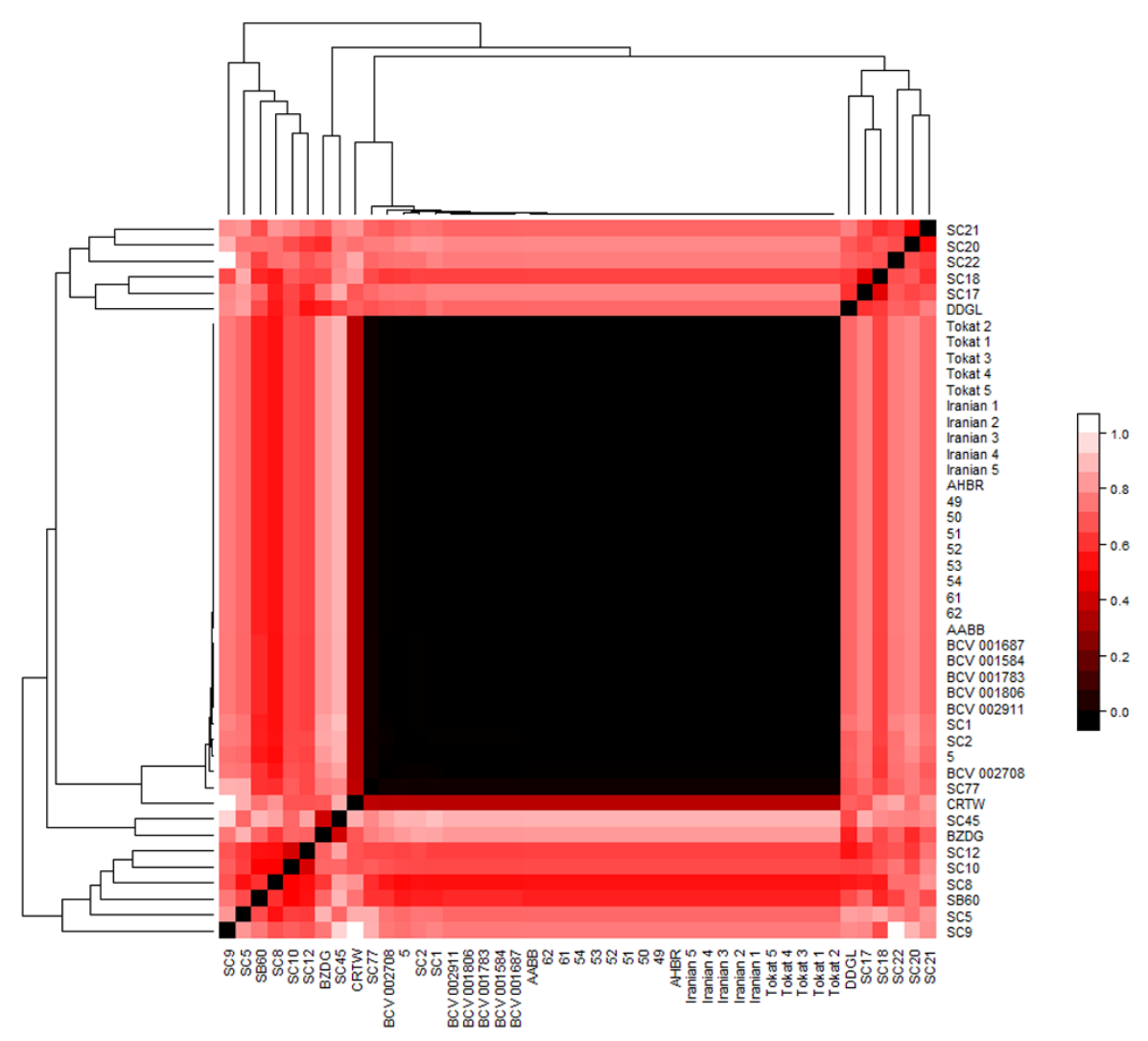

Figure 2. The heat map obtained from the analysis of iPBS-retrotransposon data.

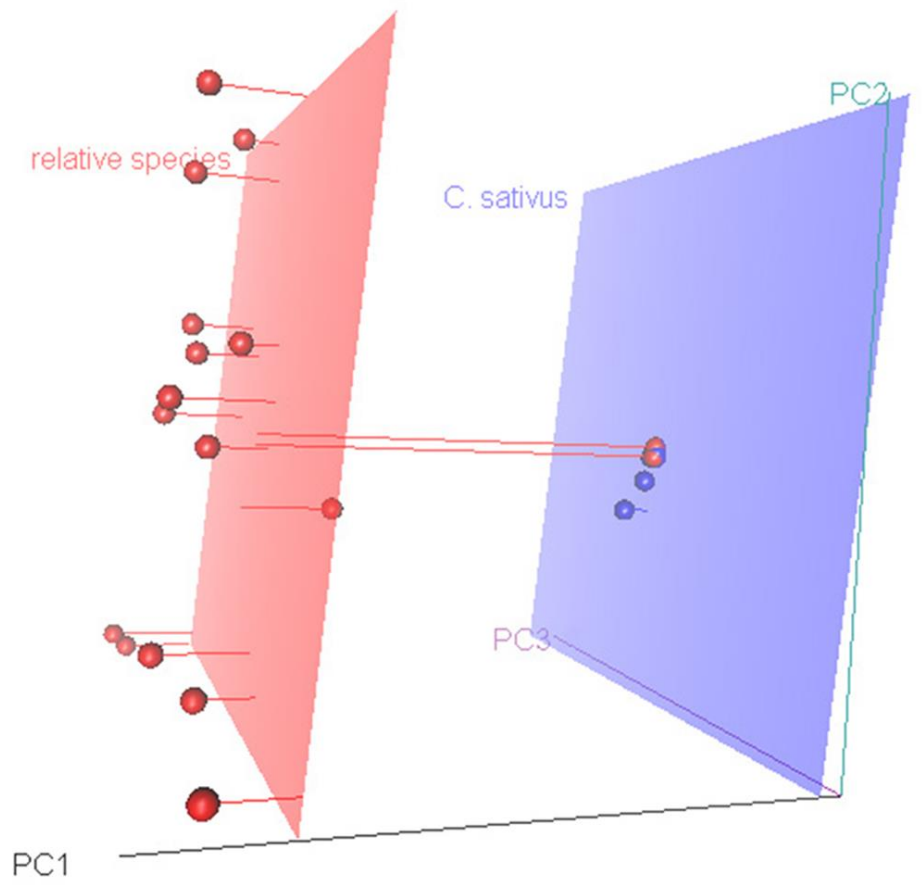

Figure 3. The relationships between the 45 saffron genotypes and its close relative species visualized by PCoA using the iPBSretrotransposon markers. 


\section{DISCUSSION}

Molecular genetic markers are advantageous for defining genetic diversity in plant breeding. Transposable elements, especially the retrotransposons, include overmuch plant genomes and replication of retrotransposons generates genetic diversity, an ability that makes them a brilliant source of molecular genetic markers (Schulman et al., 2004). These markers have been widely used in numerous studies on the genetic diversity of different plant species such as bread wheat (Gribbon et al., 1999), barley (Manninen et al., 2000), tomato (Tam et al., 2005), rice (Branco et al., 2007), Aegilops tauschii (Saeidi et al., 2008), Crocus (Alavi-Kia et al., 2008), triticale (Bento et al., 2008), Citrus and its relatives (Biswas et al., 2010), and grapevine (D’Onofrio et al., 2010). However, development of retrotransposon markers for a new plant species has certain restricting factors (Kalendar et al., 2010). Recently, Kalendar et al. (2010) proposed the iPBS-retrotransposons marker technique as a universal molecular marker based on the principle of using a single primer amplified region as a forward and reverse (Mehmood et al., 2013). These markers have been reported to amplify effectively for genetic diversity analyses (Baránek et al., 2012; Andeden et al., 2013) since the primers used for PCR can anneal to genomes that include diverse LTR sequences (Kalendar et al., 2010). However, despite being universal, to date, iPBSretrotransposons markers have only been used in the genetic diversity research of only few plants and it has not been employed to the characterization of saffron genotypes and its wild relatives.

\section{Analysis of iPBS-retrotransposon markers}

In current study, a total of 83 iPBS-retrotransposon primers were screened and 16 (19.3\%) (12-18 nt long) presented a total of 401 good, clear and polymorphic band profiles (Table 3). Similarly, Mehmood et al. (2013), Guo et al. (2014) and Nemli et al. (2015) screened 83, 41 and 83 iPBS-retrotransposon primers, respectively and selected 6, 15 and 47 primers, respectively for further analysis. These results indicate that these iPBSretrotransposon primers are not conserved among saffron, common bean, grape, guava, and other species investigated by Kalendar et al. (2010).

The average number of polymorphic iPBSretrotransposon bands found in the current study (25.1 bands) was far greater than those reported by Baránek et al. (2012) (7.1 bands), Andeden et al. (2013) (13 bands), Mehmood et al. (2013) (16.2 bands), Fang-Yong and JiHong (2014) (8.3 bands), Guo et al. (2014) (6.6 bands), and Nemli et al. (2015) (3.8 bands). This could be due to saffron having a larger genome size compared to the plant genomes studied previously. These results may also indicate that iPBS-retrotransposon primers for saffron are more conserved compared to apricot (Baránek et al., 2012), Cicer species (Andeden et al., 2013), guava (Mehmood et al., 2013), Myrica rubra (Fang-Yong and JiHong, 2014), grape (Guo et al., 2014), and common bean (Nemli et al., 2015). On the other hand, genetic diversity studies on saffron have emphasized that iPBSretrotransposon primers are more informative than other methods such as AFLP (Zubor et al., 2003), RAPD (Rubio-Moraga et al., 2009; Mir et al., 2015), SSR (Rubio-Moraga et al., 2009; Mir et al., 2015), ISSR (Rubio-Moraga et al., 2009; Mir et al., 2015), and SRAP (Babaei et al., 2014). In these studies, the iPBSretrotransposon primers generated numerous bands with a high number of copies in the plant genomes investigated. The amplified band sizes of different iPBSRetrotransposon primers used in the current study were within the range of $100-2000 \mathrm{bp}$, consistent with the results reported by Mehmood et al. (2013), Fang-Yong and Ji-Hong (2014), and Nemli et al. (2015).

The PIC value was utilized to calculate the efficiency of polymorphic loci in identifying genetic diversity (Babaei et al., 2014) and define the discriminating power of marker among genotypes (Nemli et al., 2015). In the current study, the mean PIC value was calculated as 0.85 , ranging from 0.73 (iPBS 2095) to 0.92 (iPBS 2273) (Table 3). This result indicates that iPBS 2273 was the most efficient and informative primer for discriminating among saffron genotypes. The PIC values detected in the present study were higher than those reported in previous saffron studies using other genetic marker systems. For example, Babaei et al. (2014) used SRAP markers to detect genetic diversity among saffron samples and reported the mean PIC value to be 0.15. Mir et al. (2015) used RAPD, SSR and ISSR markers to determine the molecular characterization of saffron and found the average PIC values to be 0.03 and 0.018 for RAPD and ISSR markers, respectively. On the other hand, in that study, SSR markers did not show any polymorphism. Similarly, Rubio-Moraga et al. (2009) reported that ISSR markers did not show any polymorphism in saffron. The high PIC value in the current study may be due to the large variation in the number of loci observed for iPBSRetrotransposon markers compared to AFLP, SRAP, RAPD, SSR and ISSR markers (Babaei et al., 2014; Rubio-Moraga et al., 2009; Mir et al., 2015; Zubor et al., 2003).

Since the $C$. sativus genotypes undergoes vegetative proliferation (Babaei et al., 2014), other molecular genetic markers are not able to detect or identify very low polymorphism among saffron genotypes (Babaei et al., 2014; Mir et al., 2015; Rubio-Moraga et al., 2009), iPBSretrotransposon markers are a very effective technique for the assessment of genetic diversity among saffron genotypes as confirmed by the high PIC values obtained in current study.

\section{Genetic diversity between saffron and its close relative species}

In current study, the average Nei's genetic diversity (h) and Shannon's information index (I) among saffron genotypes and its close relative species were 0.16 and 0.29 , respectively (Table 3 ), indicating a low level of differentiation. Similarly, the mean Shannon's information index for iPBS-retrotransposon markers was reported to 
be 0.12 (Yildiz et al., 2015) and 0.27 by (Mehmood et al., 2013).

The results of iPBS-retrotransposon analysis showed that the highest genetic variation was between CRTW ( $C$. cartwrightianus) and SC9 (C. mathewii). Alsayied et al. (2015) reported that $C$. cartwrightianus and $C$. mathewii were genetically distant from each other. In current study, all the $C$. sativus genotypes were in the same cluster with minimum genetic variation, but they tended to group based on their geographical origin. This showed that genotypes from the same location have no genetic variation but small genetic variation may be observed among localities (Caiola et al., 2004).

\section{Cluster and principal component analysis for the iPBS-retrotransposon markers}

The genetic diversity among saffron genotypes and its close relative species was clearly presented in the UPGMA dendrogram constructed from the DNA profile. This dendrogram placed 28 saffron genotypes and its 17 close relative species under two main clusters closely related with highly low diversity (Figures 1 and 2). The results of the principal component analysis also indicated the presence of two distinct groups based on the spatial representation of the relative genetic distances among 28 saffron samples and its 17 close relative species (Figure 3), supporting the results from the UPGMA dendrogram in that saffron genotypes had very limited genetic variation. This can be explained by the high degree of commonness in these genotypes due to the sterile and triploid nature of saffron species that vegetatively propagate by their corm, not undergoing sexual reproduction (Mir et al., 2015; Fernández, 2004).

In the UPGMA dendrogram, all the $C$. sativus genotypes $(\mathrm{n}=28), C$. cartwrightianus and some $C$. pallasii subsp. pallasii were included in the same group (Figures 1-3). C. cartwrightianus has morphological similarity to $C$. sativus and studies that performed a karyotype and morphology analysis on the genotypes allied to $C$. sativus demonstrated that $C$. cartwrightianus is one of the ancestors of $C$. sativus (Alsayied et al., 2015; Caiola et al., 2004; Larsen et al., 2015) (Alsayied et al., 2015). Similar to current results, Alsayied et al. (2015) concluded, based on the IRAP data, that $C$. sativus had minimum genetic variation and its ancestors were $C$. cartwrightianus and $C$. pallasii subsp. pallasii. These findings are also supported by the flow sorting analysis of C. sativus and its close relatives (Brandizzi and Grilli Caiola, 1998; Erol et al., 2014). AFLP and RAPD fingerprinting also revealed $C$. cartwrightianus to be the closest relative of $C$. sativus (Zubor et al., 2003; Caiola et al., 2004). In addition, the flow cytometric analysis of Crocus genotypes suggested that $C$. cartwrightianus was the most likely ancestor of $C$. sativus (Brandizzi and Grilli Caiola, 1998). In the current study, C. pallasii subsp. pallasii, C. asumanie and C. mathewii were in the second group in the UPGMA dendrogram (Figures 1-3). Although the entire $C$. sativus iPBS-retrotransposon profile was different from that of all the genotypes analyzed in current study, it was most similar to that of $C$. pallasii subsp. pallasii, C. asumanie, and C. mathewii (Figures 1-3). Erol et al. (2014) supported these findings in their flow sorting study.

The general consensus regarding a good genetic molecular marker system is that it should be polymorphic, cheap, quick, and equally distributed across the whole genome. The marker should be easy to use, be able to differentiate between genetic differences, and require a low amount of DNA and no previous knowledge of the genome (Odong et al., 2011). The findings from the present study confirm that iPBS-retrotransposon markers satisfy all these criteria for both Crocus sativus and its close relative.

\section{CONCLUSION}

iPBS-retrotransposon markers are less powerful for detection of genetic diversity in close relative species according to the average Nei's genetic diversity (h) and Shannon's information index (I).

\section{ACKNOWLEDGEMENTS}

The authors express their gratitude to University of Cukurova, Scientific Research Projects Unit for their financial support (FBA-2014-2704).

\section{LITERATURE CITED}

Abdullaev, F.I. 2002. Cancer chemopreventive and tumoricidal properties of saffron (Crocus sativus L.). Exp biol med. 227(1): 20-25.

Alavi-Kia, S., S. Mohammadi, S. Aharizad and M. Moghaddam. 2008. Analysis of genetic diversity and phylogenetic relationships in Crocus genus of Iran using interretrotransposon amplified polymorphism. Biotechnol Biotec Eq. 22 (3): 795-800.

Alsayied, N.F., J.A. Fernández, T. Schwarzacher and J. HeslopHarrison. 2015. Diversity and relationships of Crocus sativus and its relatives analysed by inter-retroelement amplified polymorphism (IRAP). Ann Bot-London. 116(3): 359-368.

Andeden, E.E., F.S. Baloch, M. Derya, B. Kilian and H. Ozkan. 2013. iPBS-Retrotransposons-based genetic diversity and relationship among wild annual Cicer species. J Plant Biochem Biot. 22(4): 453-466.

Arslan, N., A. Ozer and R. Akdemir. 2007. Cultivation of saffron (Crocus sativus L.) and effects of organic fertilizers to the flower yield. Paper read at I International Medicinal and Aromatic Plants Conference on Culinary Herbs. 826.

Babaei, S., M. Talebi, M. Bahar and H. Zeinali. 2014. Analysis of genetic diversity among saffron (Crocus sativus) accessions from different regions of Iran as revealed by SRAP markers. Sci Hortic-Amsterdam. 171: 27-31.

Baloch, F.S., A. Alsaleh, L.E.S. de Miera, R. Hatipoglu, V. Ciftci, T. Karakoy, M. Yildiz and H. Ozkan. 2015a. DNA based iPBS-retrotransposon markers for investigating the population structure of pea (Pisum sativum) germplasm from Turkey. Biochem Syst Ecol. 61: 244-252.

Baloch, F.S., M. Derya, E.E. Andeden, A. Alsaleh, G. Comertpay, B. Kilian and H. Ozkan. 2015b. Inter-primer binding site retrotransposon and inter-simple sequence repeat diversity among wild Lens species. Biochem Syst Ecol. 58: 162-168.

Baránek, M., M. Meszáros, J. Sochorová, J. Čechová and J. Raddová. 2012. Utility of retrotransposon-derived marker 
systems for differentiation of presumed clones of the apricot cultivar Velkopavlovická. Sci Hortic-Amsterdam. 143: 1-6.

Basti, A.A., E. Moshiri, A.A. Noorbala, A.H. Jamshidi, S.H. Abbasi and S. Akhondzadeh. 2007. Comparison of petal of Crocus sativus L. and fluoxetine in the treatment of depressed outpatients: a pilot double-blind randomized trial. Prog Neuro-Psychoph. 31(2): 439-442.

Bento, M., H.S. Pereira, M. Rocheta, P. Gustafson, W. Viegas and M. Silva. 2008. Polyploidization as a retraction force in plant genome evolution: sequence rearrangements in Triticale. PLoS One. 3(1): e1402.

Biswas, M.K., M. Baig, Y.J. Cheng and X.X. Deng. 2010. Retrotransposon based genetic similarity within the genus Citrus and its relatives. Genet Resour Crop Ev. 57(7): 963-972.

Branco, C. J., E.A. Vieira, G. Malone, M.M. Kopp, E. Malone, A. Bernardes, C.C. Mistura, F. I. Carvalho and C.A. Oliveira. 2007. IRAP and REMAP assessments of genetic similarity in rice. J Appl Genetics. 48(2): 107-113.

Brandizzi, F. and M. Grilli Caiola. 1998. Flow cytometric analysis of nuclear DNA in Crocus sativus and allies (Iridaceae). Plant Syst Evol. 211(3): 149-154.

Busconi, M., L. Colli, R.A. Sánchez, M. Santaella, M. D.L.M. Pascual, O. Santana, M. Roldán and J.A. Fernández. 2015. AFLP and MS-AFLP analysis of the variation within saffron crocus (Crocus sativus L.) germplasm. PLoS One. 10(4): e0123434.

Caiola, M.G., P. Caputo and R. Zanier. 2004. RAPD analysis in Crocus sativus L. accessions and related Crocus species. Biol Plantarum. 48(3): 375-380.

Castillo, R., J.A. Fernández and L. Gómez-Gómez. 2005. Implications of carotenoid biosynthetic genes in apocarotenoid formation during the stigma development of Crocus sativus and its closer relatives. Plant Physiol. 139(2): 674-689.

Comertpay, G., F. Baloch, M. Derya, E. Andeden, A. Alsaleh, H. Surek and H. Ozkan. 2015. Population structure of rice varieties used in Turkish rice breeding programs determined using simple-sequence repeat and inter-primer binding siteretrotransposon data. Genet Mol Res. 15(1).

D’Onofrio, C., G. De Lorenzis, T. Giordani, L. Natali, A. Cavallini and G. Scalabrelli. 2010. Retrotransposon-based molecular markers for grapevine species and cultivars identification. Tree Genet Genomes. 6(3): 451-466.

Doyle, J.J. J.L. Doyle. 1987. A rapid DNA isolation procedure for small quantities of fresh leaf tissue. Phytochemical Bulletin. 19:11-15.

Erol, O., H.B. Kaya, L. Sik, M. Tuna, L. Can and M.B. Tanyolac. 2014. The genus Crocus, series Crocus (Iridaceae) in Turkey and 2 East Aegean islands: a genetic approach. Turk J Biol. 38(1): 48-62.

Fang-Yong, C. and L. Ji-Hong. 2014. Germplasm genetic diversity of Myrica rubra in Zhejiang Province studied using inter-primer binding site and start codon-targeted polymorphism markers. Sci Hortic-Amsterdam. 170: 169175.

Fernández, J.A. 2004. Biology, biotechnology and biomedicine of saffron. Recent Research Developments in Plant Science. 127-159.

Fernández, J.A. 2006. Anticancer properties of saffron, Crocus sativus Linn. Advances in phytomedicine. 2: 313-330.

Frello, S. and J. Heslop-Harrison. 2000. Repetitive DNA sequences in Crocus vernus Hill (Iridaceae): the genomic organization and distribution of dispersed elements in the genus Crocus and its allies. Genome. 43(5): 902-909.

Frizzi, G., M. Miranda, C. Pantani and F. Tammaro. 2007. Allozyme differentiation in four species of the Crocus cartwrightianus group and in cultivated saffron (Crocus sativus). Biochem Syst Ecol. 35(12): 859-868.

Gailite, A. and D. Rungis. 2012. An initial investigation of the taxonomic status of Saussurea esthonica Baer ex Rupr. utilising DNA markers and sequencing. Plant Syst Evol. 298(5): 913-919.

Goldblatt, P., T.J. Davies, J.C. Manning, M. Van Der Bank and V. Savolainen. 2006. Phylogeny of Iridaceae subfamily Crocoideae based on a combined multigene plastid DNA analysis. Aliso. 22: 399-411.

Gribbon, B., S. Pearce, R. Kalendar, A. Schulman, L. Paulin, P. Jack, A. Kumar and A. Flavell. 1999. Phylogeny and transpositional activity of Ty1-copia group retrotransposons in cereal genomes. Mol Gen Genet. 261(6): 883-891.

Guo, D.L., M.X. Guo, X.G. Hou and G.H. Zhang. 2014. Molecular diversity analysis of grape varieties based on iPBS markers. Biochem Syst Ecol. 52: 27-32.

Hadizadeh, F., S. Mohajeri and M. Seifi. 2010. Extraction and purification of crocin from saffron stigmas employing a simple and efficient crystallization method. Pak J Bio Sci. 13(14): 691.

Harpke, D., A. Carta, G. Tomović, V. Ranđelović, N. Ranđelović, F. R. Blattner and L. Peruzzi. 2015. Phylogeny, karyotype evolution and taxonomy of Crocus series Verni (Iridaceae). Plant Syst Evol. 301(1): 309-325.

Harpke, D., S. Meng, T. Rutten, H. Kerndorff and F.R. Blattner. 2013. Phylogeny of Crocus (Iridaceae) based on one chloroplast and two nuclear loci: ancient hybridization and chromosome number evolution. Mol Phylogenet Evol. 66(3): 617-627.

Huson, D.H. and D. Bryant. 2006. Application of phylogenetic networks in evolutionary studies. Mol Biol Evol. 23(2): 254267.

Kafkas, S. 2006. Phylogeny, evolution and biodiversity in the genus Pistacia (Anacardiaceae). Plant genome: biodiversity and evolution 1 (part C). 525-557.

Kalendar, R., K. Antonius, P. Smýkal and A. H. Schulman. 2010. iPBS: a universal method for DNA fingerprinting and retrotransposon isolation. Theor Appl Genet. 121(8): 14191430.

Kalendar, R., A. Flavell, T. Ellis, T. Sjakste, C. Moisy and A.H. Schulman. 2011. Analysis of plant diversity with retrotransposon-based molecular markers. Heredity. 106(4): 520-530.

Kerndorff, H. and E. Pasche. 2004. Two New Taxa of the Crocus bißorus Aggregate (Liliiflorae, Iridaceae) from Turkey. Biologiezentrum Linz/Austria; download unter www.biologiezentrum.at

Kerndorff, H. and E. Pasche. 2011. Two new taxa of Crocus (Liliiflorae, Iridaceae) from Turkey: Stapfia. 95: 4-17.

Kerndorff, H., E. Pasche, F. Blattner and D. Harpke. 2013. A new species of Crocus (Liliiflorae, Iridaceae) from Turkey. Stapfia. 99: 141-144.

Kerndorff, H., E. Pasche, D. Harpke and F. Blattner. 2012. Seven new species of Crocus (Liliiflorae, Iridaceae) from Turkey. Stapfia. 97: 3-16.

Kumar, L.S. 1999. DNA markers in plant improvement: an overview. Biotechnol Adv. 17(2): 143-182.

Kumar, P., V. Gupta, A. Misra, D. Modi and B. Pandey. 2009. Potential of molecular markers in plant biotechnology. Plant Omics. 2(4): 141.

Larsen, B., J. Orabi, C. Pedersen and M. Ørgaard. 2015. Large intraspecific genetic variation within the Saffron-Crocus group (Crocus L., series Crocus; Iridaceae). Plant Syst Evol. 301(1): 425-437.

Manninen, O., R. Kalendar, J. Robinson and A. Schulman. 2000. Application of BARE-1 retrotransposon markers to the 
mapping of a major resistance gene for net blotch in barley. Mol Gen Genet. 264(3): 325-334.

Mathew, B. 1982. The Crocus, a revision of the genus Crocus BT Batsford, London.

Mehmood, A., M.J. Jaskani, S. Ahmad and R. Ahmad. 2013. Evaluation of genetic diversity in open pollinated guava by iPBS primers. Pak. J. Agri. Sci. 50(4): 591-597.

Mir, J.I., N. Ahmed, M.H. Khan, T.A. Mokhdomi, S.H. Wani, S. Bukhari, A. Asif and R.A. Qadri. 2015. Molecular characterization of saffron-potential candidates for crop improvement. Not Sci Biol. 7(1): 81.

Mondini, L., A. Noorani and M.A. Pagnotta. 2009. Assessing plant genetic diversity by molecular tools. Diversity .1(1): 19-35.

Moraga, A.R., A. Trapero-Mozos, L. Gómez-Gómez and O. Ahrazem. 2010. Intersimple sequence repeat markers for molecular characterization of Crocus cartwrightianus cv. albus. Ind Crop Prod 32(2): 147-151.

Mortazavi, S., M. Kamali Moghaddam, S. Safi and R. Salehi. 2012. Saffron petals, a by-product for dyeing of wool fibers. Prog Color Colorants Coat. 5: 75-84.

Mousavi, S.Z. and S.Z. Bathaie. 2011. Historical uses of saffron: Identifying potential new avenues for modern research. Avicenna Journal of Phytomedicine. 1(2): 57-66.

Negbi, M. 2003. Saffron: Crocus sativus L: CRC Press.

Nei, M. 1987. Molecular evolutionary genetics: Columbia university press.

Nei, M. and W.H. Li. 1979. Mathematical model for studying genetic variation in terms of restriction endonucleases. $\mathrm{P}$ Natl Acad Sci. 76(10): 5269-5273.

Nemli, S., T. Kianoosh and M.B. Tanyolac. 2015. Genetic diversity and population structure of common bean (Phaseolus vulgaris L.) accessions through retrotransposonbased interprimer binding sites (iPBSs) markers. Turk J Agric For. 39(6): 940-948.

Nørbæk, R., K. Brandt, J.K. Nielsen, M. Ørgaard and N. Jacobsen. 2002. Flower pigment composition of Crocus species and cultivars used for a chemotaxonomic investigation. Biochem Syst Ecol. 30(8): 763-791.

Ozhatay, N. 2002. Diversity of bulbous monocots in Turkey with special reference. Chromosome numbers. Pure Appl Chem. 74(4): 547-555.

Pearce, S.R., D. Li, A. Flavell, G. Harrison, J. Heslop-Harrison and A. Kumar. 1996. TheTy1-copia group retrotransposons inVicia species: copy number, sequence heterogeneity and chromosomal localisation. Mol Gen Genet. 250(3): 305-315.

Peruzzi, L. and A. Carta. 2011. Crocus ilvensis sp. nov.(sect. Crocus, Iridaceae), endemic to Elba Island (Tuscan archipelago, Italy). Nord J Bot. 29(1):0̈6-13.

Petersen, G., O. Seberg, S. Thorsøe, T. Jørgensen and B. Mathew. 2008. A phylogeny of the genus Crocus (Iridaceae) based on sequence data from five plastid regions. Taxon. 57(2): 487-499.

Pitsikas, N. and N. Sakellaridis. 2006. Crocus sativus L. extracts antagonize memory impairments in different behavioural tasks in the rat. Behav Brain Res. 173(1): 112-115.
Rohlf, F. 2000. NTSYS-pc Numerical taxonomy and multivariate analysis system, version 2.01. Setauket New York: Applied Biostatistics: Inc.

Rubio-Moraga, A., R. Castillo-López, L. Gómez-Gómez and O. Ahrazem. 2009. Saffron is a monomorphic species as revealed by RAPD, ISSR and microsatellite analyses. BMC research notes. 2(1): 1 .

Saeidi, H., M.R. Rahiminejad and J. Heslop-Harrison. 2008. Retroelement insertional polymorphisms, diversity and phylogeography within diploid, D-genome Aegilops tauschii (Triticeae, Poaceae) sub-taxa in Iran. Ann Bot-London. 101(6): 855-861.

SanMiguel, P., A. Tikhonov, Y.K. Jin and N. Motchoulskaia. 1996. Nested retrotransposons in the intergenic regions of the maize genome. Science. 274(5288): 765.

Schneider, I., H. Kerndorff and E. Pasche. 2012. Chromosome numbers of Turkish Crocus (Liliiflorae, Iridaceae) and their geographical distribution. Feddes repertorium. 123(1): 7379.

Schulman, A.H., A.J. Flavell and T.N. Ellis. 2004. The application of LTR retrotransposons as molecular markers in plants. Mobile Genetic Elements: Protocols and Genomic Applications. 145-173.

Schulman, A.H., A.J. Flavell, E. Paux and T.N. Ellis. 2012. The application of LTR retrotransposons as molecular markers in plants. Mobile Genetic Elements: Protocols and Genomic Applications. 115-153.

Seberg, O. and G. Petersen. 2009. How many loci does it take to DNA barcode a crocus? PLoS One. 4(2): e4598.

Smýkal, P., N. Bačová-Kerteszová, R. Kalendar, J. Corander, A.H. Schulman and M. Pavelek. 2011. Genetic diversity of cultivated flax (Linum usitatissimum L.) germplasm assessed by retrotransposon-based markers. Theor Appl Genet. 122(7): 1385-1397.

Tam, S.M., C. Mhiri, A. Vogelaar, M. Kerkveld, S.R. Pearce and M.A. Grandbastien. 2005. Comparative analyses of genetic diversities within tomato and pepper collections detected by retrotransposon-based SSAP, AFLP and SSR. Theor Appl Genet. 110(5): 819-831.

Verma, S. and A. Bordia. 1998. Antioxidant property of Saffron in man. Indian J Med Sci. 52(5): 205-207.

Weising, K., H. Nybom, M. Pfenninger, K. Wolff and G. Kahl. 2005. DNA fingerprinting in plants: principles, methods, and applications: CRC press.

Yildiz, M., M. Kocak and F. Baloch. 2015. Genetic bottlenecks in Turkish okra germplasm and utility of iPBS retrotransposon markers for genetic diversity assessment. Genet Mol Res. 14(3): 10588-10602.

Yuzbasioglu, S., S. Aslan and N. Ozhatay. 2015. Crocus thracicus (Iridaceae), a new species from north-western Turkey. DOI: http://dx.doi.org/10.11646/phytotaxa.197.3.4.

Zubor, A., G. Surányi, Z. Győri, G. Borbély and J. Prokisch. 2003. Molecular biological approach of the systematics of Crocus sativus L. and its allies. Paper read at I International Symposium on Saffron Biology and Biotechnology. 650. 